\title{
ESTRATEGIAS COGNITIVAS, CONTROLABILIDAD SITUACIONAL Y ESTADO AFECTIVO: EL CASO DEL PESIMISMO DEFENSIVO Y EL OPTIMISMO
}

\author{
EVARISTO FERNÁNDEZ y JOSÉ BERMÚDEZ \\ Universidad Nacional de Educación a Distancia
}

(Aceptado en febrero de 2001)

\begin{abstract}
Este estudio evalúa el papel de dos componentes del control situacional, Dificultad y Contingencia ambiental (responsividad), en la activación-interferencia de estrategias en pesimistas defensivos y optimistas, por un lado, y sus consecuencias afectivas y conductuales (nivel de rendimiento), por otro. Con este fin, utilizamos un diseño factorial 2(Grupo: pesimistas defensivos, optimistas) $\times 2$ (Dificultad: alta, baja) $\times 2$ (Contingencia: baja, alta). Con ello pretendramos estimular un nivel de control ambiental congruente o no con cada estrategia, con el fin de evaluar su influencia sobre la ansiedad, el afecto positivo-negativo y el rendimiento en una tarea de anagramas. Los resultados mostraron que los dos componentes de control situacional estudiados cumplen funciones diferentes en cuanto a la definición de la calidad del afrontamiento en pesimistas defensivos, principalmente. Concretamente, la Dificultad establece el nivel de congruencia persona (estilo valorativo)-ambiente (controlabilidad), mientras que la Contingencia actúa como moderador de esta relación. En este sentido, el estado afectivo resulta ser un adecuado indicador adaptativo del afrontamiento resultante.
\end{abstract}

Palabras clave: Activación de estrategias, congruencia persona-ambiente, control, afectividad.

\section{Cognitive strategies, situational controllability, and affective state:} the case of defensive pessimism and optimism

This study assesses the role of two components of situational control, Difficulty and environmental Contingency (responsivity), in the activation-interference of strategies in defensive pessimists and optimists, on the one hand, and their affective and behavioral (performance level) consequences, on the other. For this purpose, we use a 2 (Group: defensive pessimists, optimists) $\times 2$ (Difficulty: high, low) $\times 2$ (Contingency: low, high) factorial design. Our aim was to foster a congruent or incongruent environmental control level with each group, with the purpose of assess their influence on anxiety, positive-negative affect, and performance. The results showed that the two components of situational control have different functions in the definition of the quality of coping in defensive pessimists, principally. Specifically, Difficulty establishes the person (valorative style)-environment (controllability) congruence level, while Contingency operates as a moderator of this relation. In this sense, the affective state proves to be an adequate adaptative indicator of the resultant coping.

Key words: activation of strategies, person-environment congruence, control, affectivity.

\section{INTRODUCCIÓN}

El estudio de las estrategias cognitivas de afrontamiento ha experimentado un importante auge en los últimos años, que

Correspondencia: Evaristo Fernández, Universidad Nacional de Educación a Distancia, Facultad de Psicologia, Dpto. de Personalidad, Evaluación y Tratamiento Psicológicos, Ciudad Universitaria s/n, 28040 Madrid. Tfno. 91398 8230, Correo-e: efernandezOpsi.uned.es viene a representar un cambio de énfasis dentro del estudio de la personalidad y la motivación. En lugar de centrarse en el estudio de las disposiciones o de los motivos, o, en el otro extremo, en las secuencias conductuales moleculares, el concepto de estrategia cognitiva implica focalizarse en los procesos por los cuales la motivación, la habilidad y las disposiciones se manifiestan en la ejecución 
(Norem, 1989; Fernández, 1999). En este contexto, se entiende por estrategia cognitiva aquellos patrones de valoración, planificación, retrospección y esfuerzo que permiten traducir las metas y las creencias en conducta (Bruner, Goodnow y Austin, 1956; Showers y Cantor, 1985).

Dentro de este nivel de análisis intermedio, y refiriéndonos a un contexto de logro, se han identificado dos grupos de personas, los pesimistas defensivos y los optimistas, que se caracterizan por tener niveles de rendimiento similares a pesar de utilizar estrategias bien distintas cuando afrontan este tipo de situaciones. Por una parte, el pesimismo defensivo consiste en fijar unas expectativas bajas ante futuros resultados, a pesar de tener una experiencia pasada de éxitos (Norem y Cantor, 1986a). Estas personas se sienten muy ansiosas y experimentan un bajo control percibido antes de emprender una tarea. Sin embargo, esta valoración pesimista de la situación no se traduce en un bajo rendimiento, sino que el hecho de anticipar unos malos resultados les permite confrontar la ansiedad anticipatoriamente, produciéndose un aumento de la motivación y el esfuerzo para evitar ese tipo de resultados indeseados, así como el encauzamiento de la ansiedad en unos niveles manejables que no interfieren con la ejecución de esa tarea. El resultado es la consecución de un buen rendimiento y una valoración posterior positiva de la situación (Norem y Cantor, 1986b). Por su parte, los optimistas no anticipan unos resultados negativos; antes al contrario, tratan de distraer su atención de los aspectos de la situación (Spencer y Norem, 1996) y, en consonancia con sus éxitos pasados, fijan unas altas expectativas de rendimiento. $\mathrm{Al}$ contrario que los pesimistas defensivos, los optimistas tienden a protegerse de un fracaso sólo cuando éste ocurre, no antes, sirviéndose de la estrategia atribucional consistente en negar su responsabilidad o control sobre este tipo de resultados y de afirmarlos ante la ocurrencia de un éxito (Norem y Cantor, 1986b; 1990).

Algunos de los estudios efectuados en torno al pesimismo defensivo y el optimismo han tenido como objetivo comprobar el carácter funcional y la efectividad de las estrategias que los caracterizan, desde el punto de vista emocional y conductual (nivel de ejecución). Concretamente, el procedimiento básico de estos estudios ha consistido en inducir una serie de respuestas congruentes o no con el estilo valorativo y de afrontamiento de pesimistas defensivos y optimistas. Las variables manipuladas han sido, por un lado, el nivel y/o contenido de la focalización en la tarea, y, por otro, la funcionalidad de las expectativas.

La lógica que subyace al primer tipo de estudios es que la focalización o anticipación de los elementos negativos de la situación permite a los pesimistas defensivos manejar su ansiedad y planificar las acciones adecuadas para asegurar un buen rendimiento (Norem e Illinworth, 1993; Spencer y Norem, 1996). Por este motivo, se ha postulado, $y$ encontrado, que una interferencia con esta respuesta de focalización negativa puede entorpecer este proceso adaptativo en estos sujetos.

En el caso de los optimistas se postula todo lo contrario. Se piensa que el proceso de anticipación y focalización no forma parte de su estrategia (Norem e Illinworth, 1993; Spencer y Norem, 1996). Es decir, una vez que los optimistas han fijado unas altas expectativas de éxito, no comienzan a construir escenarios posibles a través de los cuales imaginar diversas posibilidades. De actuar así, podrían encontrar como probables diversos resultados negativos que les llevarían a un aumento de la ansiedad. Así pues, estos sujetos parecen evitar el proceso de reflexión futura con el fin de evitar, al mismo tiempo, pensar en posibilidades negativas. 
Por otro lado, en el caso de la manipulación de la funcionalidad de las expectativas, Norem y Cantor (1986b, exp. 2), encontraron que la interferencia con el beneficio de unas bajas expectativas de rendimiento, como consecuencia de la estimulación de la confianza de los pesimistas defensivos para ejecutar una futura tarea, conducía a un descontrol de la ansiedad, a una pérdida de motivación y, por tanto, a un peor rendimiento. En los optimistas, por el contrario, el estímulo de esa confianza suponía un aliciente más que les permitía mantener o aumentar sus expectativas previas de éxito, mientras que la ausencia de ese estímulo no les perjudicaba.

A partir de estos primeros estudios es posible preguntarse si la razón última por la que aquellos procedimientos estaban induciendo o no un estado congruente con cada estrategia no podía estar en un proceso más central y básico como es la valoración de controlabilidad situacional. Es decir, cuando al pesimista defensivo se le pide que adopte una estrategia congruente con su estilo habitual de valoración y afrontamiento (focalización en resultados negativos, no inducción de confianza) se le puede estar induciendo un estado inicial de falta de control que, recordemos, es lo que suele experimentar cuando entra en contacto con la situación. Esta falta de control es la que dispararía, a continuación, el resto de los mecanismos que hemos descrito anteriormente. Por el contrario, cuando a este tipo de personas se le pide que adopte una estrategia incongruente con su valoración y afrontamiento habituales (distracción o evitación cognitiva de información futura negativa, inducción de confianza) se les puede estar creando al mismo tiempo un estado de control inicial de la situación, al que no están habituados, por una parte, y que impedirá la activación de sus estrategias efectivas de adquisición de control y manejo de la ansiedad, por otra.
Con respecto a los optimistas ocurrirá algo similar. Es decir, las manipulaciones comentadas habrían estimulado un estado de no control (focalización) o de control (distracción). Sin embargo, las consecuencias de estos estados serían diferentes para estos sujetos: el primero de ellos resultaría negativo, mientras que el segundo sería positivo, a diferencia de lo que ocurría en el caso de los pesimistas defensivos.

Una manera de abordar esta cuestión consiste en proceder a la inducción de unos determinados niveles de control situacional y observar los efectos que ello ocasiona en distintos indicadores adaptativos. Un componente relevante de control situacional es la Dificultad de la tarea. Se refiere, especificamente, a la descripción de una serie de características de la situación que la hacen fácil o difícil de manejar.

Un segundo componente del control digno de ser tenido en cuenta es la Contingencia ambiental, o relación existente entre una conducta y un suceso ambiental consecuente. La expectativa que se genera a partir de la relación percibida conducta-suceso ambiental ha recibido diferentes denominaciones. Así, por ejemplo, Chapman y Skinner (1985) hablan de creencias medios-fines, y Weisz (1983) de juicios de contingencia. El denominador común a todas ellas es la percepción de que con ella es posible alterar el ambiente de tal manera que se obtengan los resultados deseados.

Teniendo en cuenta la cualidad diferencial de estos dos componentes del control, pensamos que puede resultar útil explorar las repercusiones que desde el punto de vista adaptativo pueden traer consigo la presencia de estas dos características presentes en la situación, su dificultad y su responsividad (contingencia).

Por otra parte, es necesario considerar la clase de indicadores que pueden resultar relevantes para ser utilizados como crite- 
rio de eficacia de las estrategias utilizadas por cada grupo de sujetos ante cada condición de control. Además de contemplar un indicador de tipo conductual (nivel de rendimiento), es necesario abarcar otros índices de naturaleza afectiva, y que en estudios anteriores han estado insuficientemente representados. En este sentido, en el presente estudio utilizaremos tanto una medida de ansiedad (absoluta y de cambio) como una medida de afectividad más general y comprensiva, el afecto positivo y negativo (también tomados tanto de manera absoluta como relativa).

\section{MÉTODO}

\section{Participantes}

Participaron en el experimento voluntariamente 80 sujetos $(\mathbf{4 0}$ hombres y $\mathbf{4 0}$ mujeres), estudiantes del primer ciclo de Psicología de la UNED. El rango de edad es de 18 a 44 años $(M=27,3, D T=8,3)$.

La selección se efectuó en base a las puntuaciones obtenidas en dos instrumentos, el «Life Orientation Test» (LOT; Scheier y Carver, 1985; Scheier, Carver y Bridges, 1994) y el «Optimism-Pessimism Questionnaire» (OPQ; Norem y Cantor, 1986a), a partir de una muestra inicial más amplia de 425 sujetos.

Los criterios de selección de la muestra ya han sido descritos en trabajos anteriores (Fernández, 1999; Fernández y Bermúdez, en prensa $a, b$ ). A partir de ellos se formaron dos grupos de sujetos, uno de optimistas y otro de pesimistas defensivos, equiparados en la variable sexo, de manera que habia 20 hombres y 20 mujeres en cada uno de los grupos.

\section{Diseño}

Son tres los factores a considerar en el presente estudio, todos ellos con dos nive- les: Grupo, manipulado por selección (optimistas y pesimistas defensivos), y otros dos manipulados experimentalmente: Dificultad (alta y baja) y Contingencia (baja y alta). La inteligencia se utilizó como covariable. Se trata, por tanto, de un diseño Factorial $2 \times 2 \times 2$ entre grupos.

\section{Tarea}

Elaboramos una tarea de anagramas que sería utilizada como medida de rendimiento. Estaba formada por 20 items consistentes en grupos de letras desordenadas que esconden otras tantas palabras con sentido. Estas palabras fueron seleccionadas sobre la base de su frecuencia de uso, a partir del diccionario de usos y frecuencias (Juilland y Chang-Rodríguez, 1964). Nuestro objetivo fue construir una tarea que resultara en un nivel de dificultad moderado-alto. Para ello, seleccionamos 10 palabras de frecuencia media de uso, 8 de frecuencia baja, y 2 de frecuencia alta (ver Apéndice). La duración que establecimos para esta tarea fue de 24 minutos. Como criterio de puntuación establecimos la suma de los anagramas solucionados correctamente durante ese tiempo. Véase Fernández (1999, exp. 1) para una descripción detallada de los pasos y criterios de elaboración de esta tarea.

\section{Medidas}

- Optimismo y pesimismo defensivo. Se evaluaron por medio de dos instrumentos: el Life Orientation Test (LOT) (Scheier y Carver, 1985; Scheier, Carver y Bridges, 1994), que evalúa optimismo disposicional, y utiliza una escala de respuesta tipo Likert de 5 puntos, donde $1=$ Muy en desacuerdo y $5=$ Totalmente de acuerdo, y el Optimism-Pessimism Questionnaire 
(OPQ) (Norem y Cantor, 1986a), mediante el cual evaluamos el pesimismo defensivo. La escala de respuesta es de 11 puntos, donde $1=$ En total desacuerdo y 11= Totalmente de acuerdo. En trabajos anteriores existe una descripción más detallada de estos instrumentos (Fernández, 1999; Fernández y Bermúdez, 1999).

- Ansiedad estado. La ansiedad se midió a través del State-Trait Anxiety Inventory (Spielberger, Gorsuch $\mathrm{y}$ Lushene, 1970). En este caso utilizaremos la escala estado, de 20 items. Se evaluó en tres ocasiones, antes, después de la manipulación experimental y durante la tarea. La escala de respuesta es de tipo Likert de 4 puntos ( $1=$ No, en absoluto; $2=$ Un poco; $3=$ Bastante y $4=$ Mucho). Creamos unas puntuaciones de cambio en ansiedad. Una se refería al cambio que ocurría de antes a después de la manipulación experimental, y la otra a la correspondiente al cambio desde este segundo momento al período intratarea. Estas medidas relativas de ansiedad se calcularon restando a la puntuación obtenida en ansiedad en el momento posterior la obtenida en el momento previo.

- Estado de ánimo. Se evaluó a través del "Positive and Negative Affect Schedule" (PANAS; Watson, Clark y Tellegen, 1988). Se trata de una medida compuesta por 2 escalas, una de afecto positivo y otra de afecto negativo, con 10 items cada una. Estos últimos consisten en una serie de adjetivos que describen estados de ánimo diversos. En el presente estudio se tomará como referencia temporal para evaluar el estado de ánimo el momento presente (estado). La escala de respuesta a cada ítem es de tipo Likert de 5 puntos $(1=$ Muy poco o nada en absoluto; $2=$ Un poco; $3=$ Moderadamente; $4=$ Bastante y $5=$
Extremadamente). De la misma manera que para la ansiedad, creamos puntuaciones de cambio en afecto positivo y negativo. Una de ellas se refería al cambio acontecido desde antes (nivel basal) a después de las manipulaciones experimentales, y la otra desde antes de las manipulaciones al período intratarea. Estas medidas relativas de afecto positivo y negativo se calcularon restando a las puntuaciones obtenidas en afecto durante el período intratarea las obtenidas en el momento previo a las manipulaciones.

- Dificultad percibida. Para la medida de la dificultad percibida de la tarea utilizamos un ítem ( $« E n$ qué medida considera que la tarea de anagramas será fácil o difícil»). La escala de respuesta era de 11 puntos $(0=$ Muy fácil a 10= Muy difícil). Esta medida servirá para evaluar la eficacia de la manipulación del factor Dificultad.

- Contingencia ambiental percibida. Utilizamos una medida para comprobar la eficacia de la manipulación efectuada sobre la controlabilidad de los ruidos que se iban a presentar durante la tarea ("¿En qué medida tenía la sensación de retrasar la aparición de los ruidos? »). La respuesta se realizaba en una escala de 11 puntos ( $0=$ Nada a $10=$ Muchísimo).

- Efectos de los ruidos. Elaboramos dos items para comprobar el impacto que tienen los ruidos tras el ensayo de práctica con ellos. Un primer f́tem evalúa la molestia ocasionada por los

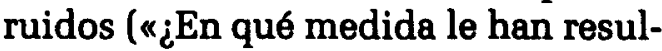
tado molestos los ruidos en sí mismos presentados durante el ensayo anterior?»), y se responde en una escala de respuesta tipo Likert de 11 puntos $(0=$ nada a $10=$ muchísimo). Un segundo ítem se refería a la creencia que tenía el sujeto acerca del efecto que podía tener en la ejecución futu- 
ra de la tarea, el hecho de poder controlar o no los ruidos. La base para contestar a este ítem era la respuesta dada a la pregunta sobre el grado de control experimentado durante la tarea de práctica con los ruidos. La formulación del ítem era distinta según el caso: "Si contestó a la pregunta número 1 de esta página con un 46 menos (con un 66 más), ¿en qué medida cree que le puede perjudicar (beneficiar) el no poder (el poder) controlar que los ruidos aparezcan cada vez más tarde?». El formato de respuesta era una escala tipo Likert de 7 puntos ( $0=$ Nada, a $6=$ Muchísimo).

- Inteligencia. Incluimos al final del experimento una breve prueba de inteligencia, destinada a controlar la posible variabilidad existente en esta variable en los diversos grupos de sujetos. Concretamente, pretendíamos evaluar la capacidad de razonamiento, dimensión estrechamente relacionada con la capacidad principal implicada en la tarea de anagramas. Nos valimos para ello del Test de Matrices progresivas de Raven, Nivel Superior (Raven, 1956). Sin embargo, no aplicamos todo el test (que sería excesivamente largo para la situación en la que nos encontrábamos), sino que seleccionamos 4 items de la serie 1 , y 12 de la serie 2, que debían ser solucionados en 16 minutos.

\section{Procedimiento}

En Fernández (1999; exp.1) puede encontrarse una exposición detallada del procedimiento empleado en esta investigación. A continuación tan solo describiremos sus aspectos más significativos.

Las sesiones experimentales fueron individuales, y tuvieron lugar en un laboratorio acondicionado especialmente para este estudio.
Tras un período de acomodación al lugar el experimentador se dirigía al sujeto y le entregaba un cuadernillo con todas las instrucciones y preguntas necesarias para el estudio. En la primera página aparecía descrito el objetivo general del experimento. A continuación comenzaba una primera parte del estudio, destinada a evaluar la ansiedad y el afecto positivo y negativo, previos todos ellos a la manipulación experimental a la que iban a ser sometidos. Estas medidas nos permitirian obtener posteriormente una medidas de cambio en estas respuestas.

Posteriormente, los sujetos eran sometidos a las distintas manipulaciones experimentales planeadas, a las que habían sido asignados al azar. Se asignaron 10 sujetos optimistas ( 5 hombres y 5 mujeres) a cada tratamiento experimental resultante de la combinación de los niveles del segundo y tercer factor: Dificultad y Contingencia. La misma asignación se efectuó con los pesimistas defensivos.

\section{Manipulaciones}

- Dificultad de la tarea (Controlabilidad objetiva): Dos recursos utilizados habitualmente en la investigación para manipular esta variable son la dificultad de la tarea y la focalización en los detalles de la tarea. En una primera fase utilizamos esta última. Elaboramos unas instrucciones en las que se comentaban diversos aspectos de la tarea de anagramas. En ellas se enfatizaban aspectos dificultosos o, por el contrario, manejables de la tarea, en función de la condición experimental de que se tratara, dificultad alta o baja, respectivamente. Cuando terminaban de leer las instrucciones comenzaba un segundo momento de la manipulación de la Dificultad. Unas instrucciones mencionaban la existencia de una tarea de 
práctica con anagramas fáciles o difíciles (según la condición de dificultad de que se tratara) destinada a familiarizarse con la tarea de anagramas posterior. Sin embargo, el objetivo principal era el de reforzar las instrucciones previas acerca de la tarea, y asegurar la inducción de unos niveles de dificultad determinados (Véase Fernández, 1999).

- Contingencia situacional (Controlabilidad subjetiva): A continuación procedimos a la manipulación de la expectativa de contingencia ambiental. Para ello utilizamos el ruido como estŕmulo externo potencialmente interferente con el rendimiento en la tarea. Todos los sujetos eran informados de que durante la ejecución de la tarea de anagramas posterior se les presentaría una serie de ruidos que podrían interferir con su rendimiento, y que uno de los objetivos del presente estudio era el de comprobar cómo el ruido afectaba a la capacidad de reacción para reducir su ocurrencia. En realidad, esta información tenía el propósito, en primer lugar, de inducir preocupación en los sujetos acerca del efecto de los ruidos y, en segundo lugar, y como consecuencia de ello, estimularles y hacer justificable a los sujetos el hecho de intentar controlar los ruidos y evitar así sus efectos interferentes. El objetivo último que perseguíamos con este tipo de información era el de posibilitar que los sujetos comprobaran directamente el grado real de efectividad que tenían sus intentos de control, mediante la realización de una tarea de práctica que vendría a reforzar la creencia de control inducida previamente por medios verbales, a los que nos referiremos más abajo.

A diferencia del control más directo u objetivo que venía representado por el nivel de dificultad de la tarea, en este caso la inducción del grado de contingencia conducta-resultado de la tarea es más indirecto y subjetivo, resultante a partir de una expectativa conducta-suceso interferente (ruido). A partir de esta expectativa era de esperar que los sujetos establecieran una generalización del nivel de control inducido sobre el ruido a una expectativa de contingencia conducta-resultado en la tarea, al sugerir mediante las instrucciones que este último se verá afectado de manera diferente en función del grado de control obtenido sobre el ruido. Procediendo de esta forma, tratábamos de reproducir la manera en que una expectativa de contingencia se forja en el medio natural, en donde previamente existe o no una expectativa de que una conducta (p.ej. el esfuerzo) ejercerá una influencia sobre los obstáculos existentes para la obtención de un buen rendimiento, lo que en última instancia redundará en la calidad final del mismo.

Y refiriéndonos ya a la manipulación de las citadas expectativas de contingencia, unas instrucciones trataban de facilitar (en el caso de la contingencia alta) o dificultar (en el caso de la contingencia baja) la posibilidad de controlar los ruidos. Se trataba así de transmitir la idea de que durante la tarea la ejecución se vería interferida en distinto grado. El contenido de las instrucciones sobre la tarea de práctica con los ruidos era variable también en función del nivel de contingencia que se tratara de inducir (véase Fernández, 1999, para una exposición detallada de las distintas instrucciones).

Cuando los sujetos informaban que estaban preparados y que habían entendido todo bien, se les decía que se pusieran los auriculares y cogieran el interruptor que supuestamente les 
permitiría manejar los ruidos. En ese momento comenzaba el ensayo práctico con los ruidos. Para ello, utilizamos dos dispositivos para la emisión de ruidos. Por una lado, un generador de ruidos, marca LETICA LE 150, cuyas características permitían seleccionar el tipo e intensidad del ruido a emitir. Conectado a él estaba un programador de estímulos, marca LETICA LE 100, que posibilitaba una emisión programada de los ruidos.

Adicionalmente utilizamos unos auriculares para la presentación de los ruidos, y un interruptor portátil (no fijo al aparato) con el cual los sujetos creían poder manejar los ruidos (ver explicación más abajo).

El aparato emitía ruidos agudos con una duración de 2 segundos, una intensidad de $90 \mathrm{~dB}$ y una frecuencia fija preestablecida por el experimentador. Del interruptor salía un cable en dirección al generador de ruidos, que en realidad no estaba conectado a él, aunque los sujetos creían que sí lo estaba. Es decir, en contra de lo que se hacía creer a los sujetos, independientemente de la acción ejercida por ellos sobre el interruptor, los ruidos aparecían según un programa de intervalos previamente fijado en función del nivel de contingencia que se quisiera inducir (Véase Fernández, 1999, para más detalles).

Finalizadas las manipulaciones experimentales, los sujetos contestaban a unas preguntas destinadas a comprobar la eficacia de la manipulación del control sobre los ruidos (ver apartado de medidas). Con respecto a la manipulación de la Dificultad, la pregunta que evaluaba la eficacia de su manipulación se presentó más adelante, mezclada con el resto de medidas del período pretarea.

Lo siguiente fue un período de descanso de 3 minutos que, en realidad, tenía como función dar tiempo a los sujetos para procesar, sobre la base de sus características personales, toda la información y experiencias recibidas hasta ese momento. Ello facilitaría la elaboración y puesta en práctica de sus estrategias habituales de evaluación y afrontamiento situacionales.

\section{Evaluación pretarea (postmanipulación)}

Transcurrido el descanso comenzaba una nueva fase del experimento, el primer período de evaluación. Se decía a los sujetos que aún faltaban unos minutos para que comenzara la tarea, y que previamente se les iba a pedir que contestaran a una serie de cuestiones. Este aviso no era algo gratuito, pues con él se pretendía activar el funcionamiento de determinadas estrategias cognitivas de afrontamiento, cuyas consecuencias esperábamos se plasmaran en la respuesta de ansiedad a evaluar a continuación (principalmente en la medida de cambio; ver apartado de medidas).

Finalizado este primer período evaluativo se anunciaba a los sujetos que el comienzo de la tarea era inminente. Procedíamos entonces a recordar algunas de las instrucciones de la tarea (ya descritas durante las manipulaciones experimentales), que mencionaban en qué iba a consistir, su duración, qué debían hacer, y el criterio de corrección que utilizaríamos.

Con respecto a los ruidos, existían dos diferencias en su emisión durante la tarea, con respecto a la forma en que se produjo durante la tarea de práctica de la manipulación. Una fue que la intensidad del ruido fue algo menor ( $80 \mathrm{~dB}$ ). La otra era el grado de control existente sobre los ruidos, que pasó a ser moderado para todos los sujetos. Al proceder de esta manera pretendíamos, por un lado, que el ruido no afectara innecesariamente al 
rendimiento $y$, por otro, mantener constantes las condiciones ambientales externas para todos los sujetos, de la misma manera que conseguíamos con la tarea principal de anagramas, que presentaba un nivel de dificultad moderada-alta para todos los sujetos.

\section{Evaluación intratarea}

Cuando habian transcurridos 12 minutos de la tarea el experimentador interrumpía el trabajo del sujeto y le pedía que contestara a algunas preguntas. Se le informaba que después volvería con la tarea, pues aún disponía de 12 minutos.

Con esta interrupción pretendíamos evaluar los cambios registrados en las variables medidas durante la etapa pretarea-postmanipulación (en el caso de la ansiedad), así como los ocurridos en las respuestas evaluadas en el período premanipulación (basal) (ansiedad y afecto positivo y negativo), una vez el sujeto ha tenido una experiencia real con la tarea. Terminado este período evaluativo se pedía a los sujetos que volvieran a la tarea. Agotados los 24 minutos el experimentador les avisaba del final de la misma.

Finalmente, después de unos minutos de descanso el experimentador aplicaba la prueba de inteligencia. Una vez terminada ésta se daba por terminada la sesión y se agradecía a los sujetos su participación.

\section{RESULTADOS}

\section{Análisis preliminares}

Una serie de análisis no revelaron efectos intra o intergrupo debidos al sexo, lo que desaconsejó su tratamiento diferenciado en el presente estudio. Asimismo, las variables independientes manipuladas no afectaron a la inteligencia, lo que permitía utilizarla como covariable en el presente estudio.

Por otra parte, los datos mostraron que los sujetos asignados a la condición de Dificultad alta valoraron la tarea como más difícil $(M=7,2, D T=1,54)$ que aquellos asignados a la de Dificultad baja $(M=2,9$; $D T=1,56), t(78)=12,34, p<0,001$. Para la Contingencia en torno a los ruidos, los datos indicaban que los sujetos asignados a la condición de contingencia alta percibían un mayor control sobre el retraso de los ruidos $(M=7,35, D T=1,35)$ que los asignados a la condición de contingencia baja $(M=1,35, D T=1,31), t(78)=20,16$, $p<0,0001$. Estos datos apoyaban la eficacia de las manipulaciones realizadas sobre la dificultad percibida de la tarea y la contingencia percibida conducta-aparición de los ruidos.

Finalmente, procedimos a la evaluación del impacto de los ruidos sobre los sujetos. En primer lugar, pudimos comprobar que el ruido previamente utilizado en la tarea de práctica era molesto para los sujetos. De esta manera, era de esperar que se encontraran motivados para intentar controlarlos y poder así comprobar en qué grado lo conseguían. Asimismo, diferentes grados de control sobre los ruidos produjeron efectos distintos sobre la percepción de beneficio (contingencia alta) o perjuicio (contingencia baja) potencial del ruido para la tarea (véase Fernández, 1999, para una exposición detallada de estos y otros análisis). Estos datos apoyaban la validez del uso del ruido en nuestro experimento como estímulo que es valorado negativamente, y cuyo control se considera positivo y su no control como negativo. A partir de aquí era posible esperar consecuencias diferenciales a raíz de la experiencia previa mantenida con él. 


\section{Análisis principales}

\section{Pretarea}

\section{Ansiedad tras la manipulación}

Apareció un efecto principal de Grupo, $F(1,71)=16,4, p<0,001$ : los pesimistas defensivos informaron de más ansiedad que los optimistas. Asimismo, un efecto principal de Dificultad, $F(1,71)=13,76$, $p<0,001$, reveló que ante una dificultad alta existra más ansiedad que ante una baja.

\section{Cambio en ansiedad}

Elaboramos una medida de cambio en ansiedad postmanipulación, a partir de los niveles basales (previos a la manipulación) de ansiedad, que tenían los siguientes valores: $M=42,62 ; D T=6,58$, para los pesimistas defensivos, y $M=$ $34,5, D T=6,32$, para los optimistas, $t(78)=4,82, p=0,001$.

Asumimos que los cambios que se produzcan en la ansiedad serán situaciona- les, aunque influidos por la actividad autorreguladora puesta en marcha en la situación, en vez de por la ansiedad basal, cuyo efecto queda controlado, no afectando, por ello, a la medida de cambio en ansiedad que estamos considerando.

Con este tipo de medida es posible comprobar si las estrategias de los pesimistas y optimistas ejercen su influencia desde el punto de vista afectivo, dándose muestras de un encauzamiento de la ansiedad si ésta disminuye, no aumenta o lo hace mínimamente.

No apareció un efecto principal de Grupo: los pesimistas, en general; han conseguido controlar su nivel de ansiedad, cuyo aumento no se sitúa por encima del que ha tenido lugar para los optimistas.

$\mathrm{Si}$ apareció un efecto principal de Dificultad, aunque sólo era marginalmente significativo $F(1,75)=3,03, p=0,09$ : dificultad alta = aumento de la ansiedad; dificultad baja $=$ decremento (en el caso de los pesimistas) o ausencia de cambio (en el caso de los optimistas (Tabla 1).

Tabla 1. Descriptivos [(Media y Desviación típica ()] de las variables afectivas y del rendimiento en función de Grupo y Controlabilidad situacional [Dificultad alta (DA), Dificultad baja (DB), Contingencia baja (CB) y Contingencia alta (CA)]: fases de postmanipulación (pretarea) e intratarea (ejecución).

\begin{tabular}{lrrrrrrrr}
\hline & \multicolumn{4}{c}{ Optimistas } & \multicolumn{4}{c}{ Pesimistas defensivos } \\
& DA & DB & CB & CA & DA & DB & CB & CA \\
\hline Postmanipulación & & & & & & & & \\
Ansiedad & 39,1 & 32,8 & 36,4 & 35,5 & 47,3 & 40,7 & 45,9 & 42,1 \\
& $(6,2)$ & $(5,5)$ & $(8,1)$ & $(6,1)$ & $(8,0)$ & $(9,7)$ & $(10,7)$ & $(7,7)$ \\
Cambio ansiedad & 2,7 & 0,15 & 2,6 & 0,30 & 3,6 & $-0,70$ & 3,60 & $-0,75$ \\
& $(4,8)$ & $(3,1)$ & $(3,5)$ & $(4,9)$ & $(8,8)$ & $(5,3)$ & $(7,4)$ & $(7,1)$ \\
Intratarea & & & & & & & & \\
Ansiedad & 39,4 & 36,5 & 37,2 & 38,7 & 44,9 & 47,2 & 45,6 & 43,6 \\
& $(7,6)$ & $(6,4)$ & $(7,6)$ & $(7,8)$ & $(7,4)$ & $(7,1)$ & $(7,0)$ & $(6,9)$ \\
Cambio ansiedad & 0,30 & 3,7 & 0,80 & 3,25 & $-2,4$ & 6,5 & 2,6 & 1,4 \\
& $(3,9)$ & $(4,8)$ & $(6,2)$ & $(7,3)$ & $(4,5)$ & $(4,2)$ & $(8,2)$ & $(8,4)$ \\
Afecto positivo & 32,5 & 33,1 & 32,8 & 32,8 & 30,7 & 29,0 & 29,9 & 29,8 \\
& $(4,6)$ & $(3,2)$ & $(4,2)$ & $(3,8)$ & $(4,8)$ & $(5,4)$ & $(5,2)$ & $(4,6)$ \\
Cambio afecto positivo & 0,40 & 1,5 & 0,80 & 1,15 & 0,80 & $-1,4$ & $-0,25$ & $-0,65$ \\
& $(3,3)$ & $(2,9)$ & $(3,0)$ & $(3,3)$ & $(4,3)$ & $(5,8)$ & $(4,5)$ & $(5,8)$ \\
Afecto negativo & 14,2 & 12,8 & 12,7 & 14,3 & 18,8 & 18,9 & 20,4 & 17,3 \\
& $(5,8)$ & $(3,6)$ & $(3,1)$ & $(6,1)$ & $(7,7)$ & $(5,2)$ & $(6,1)$ & $(6,7)$ \\
Cambio afecto negativo & 0,55 & 1,35 & $-0,20$ & 2,10 & 0,80 & 3,25 & 2,95 & 1,10 \\
& $(5,06)$ & $(3,54)$ & $(4,16)$ & $(4,6)$ & $(4,06)$ & $(3,9)$ & $(3,7)$ & $(4,7)$ \\
\hline Rendimiento & 14,2 & 15,5 & 15,8 & 14,4 & 15,8 & 15,6 & 15,5 & 16,0 \\
& $(3,34)$ & $(3,7)$ & $(3,3)$ & $(3,8)$ & $(2,8)$ & $(3,4)$ & $(3,2)$ & $(2,9)$ \\
\hline
\end{tabular}


Tabla 2. Descriptivos [(Media y Desviación típica ()] de las variables afectivas y del rendimiento en función de Grupo (Optimistas, Pesimistas defensivos), Dificultad [alta (DA), baja (DB)] y Contingencia [baja (CB), alta (CA)]: fases de postmanipulación (pretarea) e intratarea (ejecución),

\begin{tabular}{|c|c|c|c|c|c|c|c|c|}
\hline & \multirow{2}{*}{\multicolumn{4}{|c|}{ Optimistas }} & \multirow{2}{*}{\multicolumn{4}{|c|}{ Pesimistas defensivos }} \\
\hline & & & & & & & & \\
\hline & CB & CA & $\mathrm{CB}$ & $\mathrm{CA}$ & $\mathrm{CB}$ & CA & CB & CA \\
\hline \multicolumn{9}{|l|}{ Postmanipulación } \\
\hline Ansiedad & $\begin{array}{r}41,5 \\
(8,4)\end{array}$ & $\begin{array}{c}36,7 \\
(6,7)\end{array}$ & $\begin{array}{c}31,3 \\
(3,2)\end{array}$ & $\begin{array}{r}34,3 \\
(7,17)\end{array}$ & $\begin{array}{r}50,8 \\
(8,0)\end{array}$ & $\begin{array}{l}43,9 \\
(6,6)\end{array}$ & $\begin{array}{r}41,1 \\
(9,17)\end{array}$ & $\begin{array}{r}40,4 \\
(8,63)\end{array}$ \\
\hline Cambio ansiedad & $\begin{array}{r}4,3 \\
(3,5)\end{array}$ & $\begin{array}{r}1,2 \\
(6,3)\end{array}$ & $\begin{array}{r}0,90 \\
(4,1)\end{array}$ & $\begin{array}{r}-0,60 \\
(2,9)\end{array}$ & $\begin{array}{r}8,0 \\
(6,8)\end{array}$ & $\begin{array}{r}-0,90 \\
(8,6)\end{array}$ & $\begin{array}{r}-0,80 \\
(5,2)\end{array}$ & $\begin{array}{r}-0,60 \\
(5,7)\end{array}$ \\
\hline \multicolumn{9}{|l|}{ Intratarea } \\
\hline Ansiedad & $\begin{array}{c}39,6 \\
(9,1)\end{array}$ & $\begin{array}{r}39,2 \\
(10,8)\end{array}$ & $\begin{array}{r}34,8 \\
(5,16)\end{array}$ & $\begin{array}{l}38,3 \\
(9,6)\end{array}$ & $\begin{array}{r}48,4 \\
(10,3)\end{array}$ & $\begin{array}{l}41,5 \\
(8,5)\end{array}$ & $\begin{array}{r}48,8 \\
(10,1)\end{array}$ & $\begin{array}{l}45,7 \\
(9,2)\end{array}$ \\
\hline Cambio ansiedad & $\begin{array}{r}-1,9 \\
(6,5)\end{array}$ & $\begin{array}{r}2,5 \\
(9,4)\end{array}$ & $\begin{array}{r}3,5 \\
(4,9)\end{array}$ & $\begin{array}{r}4,0 \\
(4,9)\end{array}$ & $\begin{array}{r}-2,4 \\
(9,8)\end{array}$ & $\begin{array}{r}-2,4 \\
(6,8)\end{array}$ & $\begin{array}{r}7,7 \\
(8,5)\end{array}$ & $\begin{array}{r}5,3 \\
(8,3)\end{array}$ \\
\hline Afecto positivo. & $\begin{array}{l}33,1 \\
(5,0)\end{array}$ & $\begin{array}{r}31,9 \\
(4,5)\end{array}$ & $\begin{array}{l}32,5 \\
(3,6)\end{array}$ & $\begin{array}{l}33,7 \\
(2,9)\end{array}$ & $\begin{array}{l}28,7 \\
(4,9)\end{array}$ & $\begin{array}{l}32,7 \\
(3,8)\end{array}$ & $\begin{array}{l}31,1 \\
(4,6)\end{array}$ & $\begin{array}{l}26,9 \\
(4,4)\end{array}$ \\
\hline Cambio afecto positivo & $\begin{array}{r}0,50 \\
(3,5)\end{array}$ & $\begin{array}{r}0,30 \\
(3,2)\end{array}$ & $\begin{array}{r}1,1 \\
(2,7)\end{array}$ & $\begin{array}{r}2,0 \\
(3,3)\end{array}$ & $\begin{array}{r}0,10 \\
(4,7)\end{array}$ & $\begin{array}{c}0,90 \\
(4,1)\end{array}$ & $\begin{array}{r}-0,60 \\
(4,5)\end{array}$ & $\begin{array}{r}-2,2 \\
(6,1)\end{array}$ \\
\hline Afecto negativo & $\begin{array}{l}13,7 \\
(3,7)\end{array}$ & $\begin{array}{l}14,7 \\
(7,6)\end{array}$ & $\begin{array}{l}11,7 \\
(1,9)\end{array}$ & $\begin{array}{l}14,0 \\
(4,5)\end{array}$ & $\begin{array}{l}21,3 \\
(6,3)\end{array}$ & $\begin{array}{r}16,4 \\
(8,4)\end{array}$ & $\begin{array}{l}18,2 \\
(4,5)\end{array}$ & $\begin{array}{l}19,6 \\
(6,0)\end{array}$ \\
\hline Cambio afecto negativo & $\begin{array}{r}-1,6 \\
(5,3)\end{array}$ & $\begin{array}{r}2,7 \\
(4,5)\end{array}$ & $\begin{array}{r}1,2 \\
(2,1)\end{array}$ & $\begin{array}{r}1,5 \\
(4,7)\end{array}$ & $\begin{array}{r}2,9 \\
(3,9)\end{array}$ & $\begin{array}{r}-1,3 \\
(4,1)\end{array}$ & $\begin{array}{r}3,0 \\
(3,7)\end{array}$ & $\begin{array}{r}3,5 \\
(4,3)\end{array}$ \\
\hline Rendimiento & $\begin{array}{l}14,6 \\
(3,2)\end{array}$ & $\begin{array}{l}14,0 \\
(3,6)\end{array}$ & $\begin{array}{l}16,1 \\
(3,1)\end{array}$ & $\begin{array}{l}14,9 \\
(3,1)\end{array}$ & $\begin{array}{l}15,2 \\
(3,1)\end{array}$ & $\begin{array}{l}16,5 \\
(2,5)\end{array}$ & $\begin{array}{l}15,8 \\
(3,5)\end{array}$ & $\begin{array}{r}15,5 \\
(3,4)\end{array}$ \\
\hline
\end{tabular}

Por otra parte, una exploración «a posteriori» revelo la existencia de un contraste significativo, $F(1,18)=6,53, p<0,05$, en el contexto de la interacción entre Grupo, Dificultad y Contingencia, que resulto marginalmente significativa, $F(1,71)=2,31, p=$ 0,12: en el grupo de pesimistas defensivos, dificultad alta, contingencia baja = aumento de ansiedad ( $p<0,01)$ (ver Tabla 2).

\section{Intratarea}

\section{Ansiedad}

Como era de esperar para esta medida absoluta, apareció un efecto principal de Grupo, $F(1,71)=15,94, p=0,001$ : los pesimistas defensivos experimentaron más ansiedad que los optimistas.

\section{Cambio en ansiedad}

Esta medida de cambio nos puede servir, como ocurría en la etapa pretarea, de indicador de que la ansiedad sigue sien- do encauzada por las estrategias defensivas de los pesimistas, disminuyendo, no aumentando o haciéndolo mínimamente, de tal manera que no supere el incremento que se ocasione en los optimistas.

Apareció un efecto principal de Dificultad, $F(1,71)=13,02, p=0,001$ : ante una dificultad baja se produjo un aumento de la ansiedad $(M=5,13)$, mientras que ante una dificultad alta tuvo lugar un ligero decremento $(M=-1,05)$.

Apareció una interacción marginalmente significativa de Grupo y Dificultad, $F(1,71)=2,84, p=0,09$, que venía explicada por los siguientes contrastes (ver Tabla 1): 1) $F(1,38)=16,6, p<0,001$ : en el grupo de pesimistas defensivos, una dificultad baja = aumento de la ansiedad $(p<0,001)$, ante una dificultad alta $=$ disminución de la ansiedad $(p<0,01) ; 2)$ en el grupo de optimistas se encontró la misma tendencia, aunque la diferencia fue menor, $F(1,38)=7,64, p<0,01 ; 3) F(1,38)=$ $3,92, p=0,05$ : en el grupo de dificultad 
baja, pesimistas defensivos = más aumento de ansiedad que en optimistas.

\section{Afecto positivo}

Apareció un efecto principal de Grupo, $F(1,71)=6,34, p<0,05$ : los optimistas experimentaron más afecto positivo que los pesimistas defensivos.

Se encontró una interacción entre Grupo, Dificultad y Contingencia, $F(1,71)=$ $4,78, p<0,05$, que venía explicada por los siguientes contrastes (ver Tabla 2): 1) $F(1,18)=7,05, p<0,01$ : en el grupo de pesimistas defensivos y una contingencia alta, una dificultad alta = más afecto positivo que ante una dificultad baja; 2 ) $F(1,18)=5,28, p<0,05$ : en el grupo de pesimistas y una dificultad alta, contingencia alta = más afecto positivo que ante una contingencia baja; 3 ) en el grupo de pesimistas y una dificultad baja, la tendencia se invertía, $F(1,18)=6,40, p=0,01$; 4) $F(1,18)=9,72, p<0,01$ : ante una contingencia alta y una dificultad baja, optimistas $=$ más afecto positivo que en pesimistas; 5) $F(1,18)=9,05, p<0,01$ : ante una contingencia baja y una dificultad alta, optimistas $=$ más afecto positivo que pesimistas.

En resumen, los datos indican que la dificultad alta, al contrario que la baja, produce un bienestar psicológico mayor en los pesimistas, pero sólo en el contexto de una contingencia alta. Si ésta es baja, la dificultad alta no ejerce ese papel protector sobre estos sujetos. Por su parte, los optimistas parecen dejarse influir menos por las diferencias en el nivel de control, informando sentirse igualmente bien en todas las condiciones.

\section{Cambio en afecto positivo}

$\mathrm{Al}$ igual que las medidas de cambio en ansiedad, la medida ajustada de afecto positivo (y negativo) puede utilizarse como un indicador del grado en que los pesimistas controlan su estado emocional a medida que transcurre el afrontamiento de la situación. El afecto positivo basal (previo a la manipulación) fue prácticamente idéntico en los pesimistas defensivos y en los optimistas $(M=30,3, D T=$ 4,73; $M=31,82, D T=4,61$, respectivamente, $t(78)=1,46, p=0,15$

No hubo ningún efecto principal de Grupo. Sí apareció una interacción entre Grupo y Dificultad, que resultó marginalmente significativa, $F(1,71)=3,02, p=$ 0,08 , que venía explicada por un único contraste (ver Tabla 1): $F(1,38)=4,02, p=$ 0,05 , ante una dificultad baja, pesimistas defensivos $=$ decremento no significativo del afecto positivo; optimistas = aumento significativo $(p<0,05)$.

\section{Afecto negativo}

Apareció un efecto principal de Grupo, $F(1,71)=17,12, p<0,001$ : los pesimistas defensivos informaron de un afecto negativo mayor que los optimistas.

Surgió también una interacción entre Grupo y Contingencia, $F(1,71)=3,87$, $p=0,05$, explicada por un único contraste, $F(1,38)=26,07, p<0,001$ (ver Tabla 1 ): ante un contingencia baja, pesimistas $=$ mayor afecto negativo.

\section{Cambio en afecto negativo}

El nivel de afecto negativo basal, sobre el que calculamos el índice de cambio para el afecto negativo, fue significativamente mayor en pesimistas defensivos ( $M=16,85$, $D T=4,02)$ que en optimistas $(M=12,58$, $D T=3,93), t(78)=4,12, p<0,001$.

No hubo efecto principal de Grupo para esta medida $(F<1)$. De manera similar a lo ocurrido con el afecto positivo, los pesimistas defensivos parecen haber evitado, en este caso, un aumento descontrolado de su emocionalidad negativa.

Por otro lado, apareció una interacción de Grupo, Dificultad y Contingencia, 
$F(1,71)=3,88, p=0,05$, que venía explicada por dos contrastes (ver Tabla 2): 1) $F(1,18)=5,93, p<0,05$ : en el grupo de pesimistas defensivos y una contingencia alta, dificultad alta = disminución no significativa de afecto negativo; dificultad baja = aumento del afecto negativo ( $p<0,05)$; 2) $F(1,18)=4,02, p=0,05$ : en el grupo de pesimistas defensivos y dificultad alta, contingencia baja $=$ aumento del afecto negativo ( $\mathrm{p}=.11$ ); contingencia alta $=$ disminución del afecto negativo $(p>$ $0,15)$.

En la misma línea de lo sucedido en la medida absoluta de afecto positivo, la condición de dificultad alta resulta positiva para los pesimistas defensivos sólo si se encuentran ante una contingencia alta. Vuelve a aparecer, por tanto, la idoneidad de la combinación Dificultad alta-Contingencia alta en los pesimistas defensivos, que, en este caso, les permite controlar la cantidad de afecto negativo que experimentan.

\section{Rendimiento}

No apareció un efecto principal significativo de Grupo para el número de aciertos. En general, los pesimistas tienen un mismo rendimiento que los optimistas $(M=15,75 ; M=15,13$, respectivamente). No obstante, pudo apreciarse una ligera desventaja en el caso de los optimistas ante una dificultad alta, en comparación con los pesimistas de su misma condición y los optimistas ante una dificultad baja, aunque estas diferencias no llegaron a ser significativas ( $p s>0,10$ ). Por otro lado, en contra de lo que cabría esperar, no aparecieron diferencias intragrupo en los pesimistas defensivos en función del nivel de control manipulado: los pesimistas ante un control alto no salieron perjudicados en cuanto a su nivel de rendimiento.

\section{DISCUSIÓN}

La consideración, por un lado, de dos componentes del control situacional, la Dificultad y la Contingencia o responsividad ambiental, $y$, por otro, de una medida de afecto positivo-negativo, ha hecho posible una comprensión más profunda del funcionamiento de las estrategias de pesimistas defensivos y optimistas.

Un primer grupo de resultados que merece ser comentado se refiere a los niveles generales de afectividad presentes en pesimistas defensivos y optimistas, tomados globalmente. Con respecto a las medidas absolutas de ansiedad $y$, durante la ejecución, también de afectividad general, los pesimistas informaron de un estado afectivo más negativo que los optimistas, como era de esperar. En el caso de la ansiedad y el afecto negativo esto estaba justificado, en parte, por los mayores niveles absolutos basales (premanipulación) existentes en los pesimistas. En el caso del afecto positivo, por el contrario, los niveles iniciales eran similares en ambos grupos de sujetos, por lo que las diferencias (incluso absolutas) aparecidas durante la tarea fueron debidas a la dinámica establecida tras el período de manipulación y durante la ejecución. Estos resultados parecen sugerir una independencia en la experiencia de afecto positivo y negativo en pesimistas defensivos, independencia ya señalada para la población general por Watson et al. (1988). Asimismo, suponen que si bien las diferencias existentes en autoestima, autoeficacia, ansiedad y pesimismo (todos ellos disposicionales), entre pesimistas defensivos y optimistas (Fernández, 1999) pueden dar cuenta de una mayor experiencia de afecto negativo en los primeros, aquellas no parecen afectar diferencialmente a la disponibilidad a experimentar emociones positivas (interés, orgullo, entusiasmo, energía, etc.) en los pesimistas 
defensivos en ausencia de una situación inmediata a afrontar y, como veremos después, tampoco en aquellos que no se ven interferidos en el uso de sus estrategias cuando sí deben afrontar tal situación: los asignados a una dificultad alta.

Un segundo grupo de resultados exige dar un paso más y contemplar los patrones afectivos existentes para ambos grupos de sujetos en interacción con los niveles de controlabilidad presentes en la situación. Ha sido posible constatar la existencia de diferentes patrones de control de la ansiedad en estos grupos de sujetos, pero también (esto es lo novedoso) de un grupo más amplio de emociones, positivas y negativas. Concretamente, en el caso de los pesimistas defensivos DB o incongruentes tenía lugar un aumento de la ansiedad y una disminución del afecto positivo durante la ejecución, después de una primera disminución de la ansiedad tras la experiencia de un estado de alto control (Dificultad baja o condición interferente). Por el contrario, en los pesimistas defensivos ante una Dificultad alta (Control bajo) se producía, durante la tarea, una disminución de la ansiedad y un mantenimiento del afecto positivo. Estos datos vienen a demostrar la funcionalidad de una característica de controlabilidad situacional (la dificultad para afrontar la situación) como elemento activador diferencial de estrategias en estos sujetos.

Por otro lado, al contrario de lo que ocurría con los pesimistas defensivos, una dificultad baja resultó ser positiva para los optimistas. No obstante, durante la tarea se producía en estos sujetos una cierta reacción a la desconfirmación de expectativas (al tratarse de una tarea algo más difícil de lo sugerido en un principio por la manipulación) representada por un aumento significativo de la ansiedad (aunque menor que en los pesimistas). Esto no suponía, sin embargo, la experiencia de una mayor ansiedad absoluta, produciéndose incluso un aumento de su afecto positivo.

Por otro lado, si una dificultad baja parecía beneficiar a los optimistas, una dificultad alta, por el contrario, les perjudicaba, llevándoles a experimentar cierta elevación de sus ansiedad, aunque sólo inicialmente. Efectivamente, este perjuicio debe entenderse meramente como una reacción lógica a la presencia en la situación de indicadores amenazantes (alta dificultad), pues durante la ejecución tiene lugar un mantenimiento de sus niveles afectivos, al contrario de lo que ocurría en los pesimistas interferidos (DB). Aquellos sujetos, posiblemente gracias a sus recursos personales (autoestima, optimismo, etc.), no presentes en los pesimistas (Fernández y Bermúdez, 1999), consiguieron remontar las dificultades iniciales y encauzar sus respuestas afectivas.

Estos resultados son coherentes con los obtenidos por estudios previos (Fernández, 1999; Fernández y Bermúdez, en prens $a, a, b)$, en donde la Dificultad introdujo diferentes patrones de reacción en pesimistas defensivos, principalmente, $y$ en optimistas, en lo que se refiere a diversos elementos definitorios de las estrategias de cada grupo de sujetos (niveles de expectativas, focalización en la situación), lo que trajo consecuencias en el nivel adaptativo logrado por los diferentes sujetos.

Teniendo en cuenta toda esta evidencia, a partir de la hipotesis de congruencia de Evans, Shapiro y Lewis (1993) y de Litt (1988), según la cual para que la existencia de control sobre el medio sea aceptada por la persona y no resulte amenazante para ella, el nivel de control debe ser congruente con su estilo preferido de valoración y afrontamiento, es posible establecer una configuración específica con respecto a distintos grados de congruencia persona-situación, que venga definida, concretamente, por el grupo de 
sujetos estudiados (pesimistas defensivos y optimistas), por un lado, y por los niveles de Dificultad situacional manipulados (alta y baja), por otro. De esta manera una Dificultad alta (DA) (bajo control) sitúa a los pesimistas en un estado de congruencia, pues un bajo control situacional es coherente con su estilo valorativo, y activa la puesta en marcha de sus estrategias habituales de afrontamiento, mientras que una Dificultad baja (DB) (alto control) se consideraría incongruente con su estilo valorativo, al interferir con esas estrategias.

Por su parte, en el caso de los optimistas, aunque no es posible hablar de congruencia-incongruencia en términos estrictos, pues estos sujetos muestran una mayor flexibilidad en su afrontamiento con el medio (y, por tanto, una menor vinculación a características situacionales), sí puede perfilarse para ellos unas condiciones de preferencia por un tipo de situación u otro. En este caso es una Dificultad baja o control alto la cualidad más idónea (aunque no determinante) para estos sujetos.

Hasta este momento hemos contemplado la funcionalidad de uno de los componentes de la controlabilidad situacional, la Dificultad. Veamos a continuación la contribución de la contingencia ambiental, esto es, la presencia-ausencia de una conducta que trae como consecuencia cambios deseados en el medio o la evitación de los no deseados, y, en última instancia, la creación de una expectativa de contingencia conducta-resultado en la tarea. Este es un aspecto central de nuestro trabajo, y para abordarlo debemos centrar nuestra atención en los resultados referentes a las interacciones de segundo orden protagonizadas por Grupo, Dificultad y Contingencia.

Las interacciones de tres factores aparecidas para las medidas de afecto positivo y de cambio en afecto negativo (así como otros contrastes significativos rele- vantes para las medidas de ansiedad y de cambio en ansiedad) ponían de manifiesto que la mejor combinación de niveles de control en el caso de los pesimistas defensivos era una Dificultad alta y una Contingencia alta. Es decir, los pesimistas congruentes (DA) que creyeron poder controlar la aparición de los ruidos interferentes durante la tarea y, por tanto, se forjaron una expectativa de control sobre los resultados en esa tarea, experimentaron el mismo nivel de afecto positivo que los optimistas, y mantuvieron, como ellos, sus niveles de afecto negativo, al contrario de lo que ocurría con los pesimistas incongruentes (DB), que no se vieron beneficiados por tener esa creencia de control sobre un suceso ambiental negativo. Cuando la contingencia era baja, sin embargo, tanto los pesimistas congruentes como los incongruentes se vieron perjudicados desde el punto de vista emocional. Este patrón de resultados es coherente con el hallado por Fernández (1999) para la dificultad percibida intratarea. En este caso, los pesimistas congruentes o DA veían la tarea, durante el período de ejecución, como más fácil que los incongruentes o $\mathrm{DB}$ e igual de fácil que los optimistas únicamente si la contingencia ambiental era alta.

Estos datos nos vuelven a indicar, desde otra perspectiva, que en los pesimistas defensivos la congruencia o no viene definida por el nivel de dificultad de la tarea (controlabilidad objetiva), y no por el existente en la contingencia ambiental (controlabilidad subjetiva o indirecta). En este sentido puede recordarse que en la interacción existente entre Grupo y Contingencia para el afecto negativo una contingencia baja (un nivel bajo de control) perjudicaba a los pesimistas defensivos, mientras que una dificultad alta (también indicando un bajo control) les beneficiaba. La contingencia ambiental, por tanto, actuaría como un moderador del efecto de la dificultad, o dicho de 
otro modo, del pesimismo defensivo congruente (Dificultad alta) y del incongruente (Dificultad baja). En el caso de los optimistas, por el contrario, los niveles de contingencia no ejercían tal función moderadora.

El papel moderador de la percepción de contingencia ambiental ha sido hallado también en otros estudios (Conway y Terry, 1992; Forsythe y Compas, 1987; Folkman, Lazarus, Dunkel-Schetter, De Longis y Gruen, 1986), que fracasan a la hora de encontrar datos a favor de un efecto directo sobre diversas medidas de adaptación, aunque en algún caso existen pruebas de que la valoración de un suceso como incontrolable se asocie, por ejemplo, con depresión (Thoits, 1983). Como Conway y Terry (1992) señalan, la naturaleza equívoca de los datos relacionando la valoración de contingencia situacional con bienestar puede depender del hecho de que la valoración de controlabilidad de un suceso actúe principalmente como un moderador de la efectividad de diferentes estrategias de afrontamiento, más que como un determinante directo de los resultados adaptativos. En nuestro estudio este efecto se manifest 6 claramente cuando la alta contingencia ambiental facilitó las estrategias de afrontamiento coherentes con el pesimismo defensivo, activadas previamente por una dificultad alta. Por el contrario, cuando los pesimistas defensivos no utilizaron unas estrategias congruentes el efecto moderador de la contingencia fue negativo. Es decir, debe existir previamente la utilización de unas estrategias coherentes y eficaces, viniendo la controlabilidad ambiental (responsividad, contingencia) simplemente a complementar esa influencia.

Una implicación importante de todos estos resultados es que todas las cualidades de un bajo control no parecen cumplir con la misma función, y no todas resultan perjudiciales para los pesimistas defensivos. Estos sujetos necesitan valorar la situación negativamente, como algo difícil de afrontar, en orden a activar sus estrategias protectoras, pero al mismo tiempo necesitan percibir que con su conducta pueden controlar la aparición de un resultado (en nuestro caso, control de un suceso ambiental que, en última instancia, maximiza un buen rendimiento). Esto sólo es posible detectarlo cuando se contempla el control ambiental de manera multidimensional, tal y como hemos procedido en el presente estudio.

Por otra parte, el hecho de que en los optimistas el nivel de contingencia no moderara el efecto de la Dificultad es coherente con la idea (apuntada anteriormente) de que en estos sujetos los niveles de Dificultad no son determinantes en la configuración de la cualidad de su afrontamiento, como sí ocurría en el caso de los pesimistas defensivos. Es muy probable que en el caso de los optimistas sean sus recursos personales de carácter disposicional los que jueguen un papel más relevante al respecto.

Finalmente, un último punto que debemos considerar se refiere a lo ocurrido con el nivel de rendimiento alcanzado por los diferentes grupos de sujetos. En un principio cabría esperar diferencias en la calidad de la ejecución sobre la base de diferentes niveles de manejo de la ansiedad y de afectividad general. Concretamente, los pesimistas DB o interferidos, principalmente, y los optimistas DA, deberían haber sufrido un deterioro en su nivel de rendimiento, como consecuencia de la inestabilidad emocional (aumento de la ansiedad-afecto negativo) experimentada en distintos momentos del proceso autorregulador. ¿Qué pudo ocurrir, pues? En el caso de los optimistas DA, si bien existía una ligera desventaja en el número de anagramas resueltos, con respecto a los pesimistas DA o congruentes y los optimistas DB, esa desventaja no fue importante. Como ya indicá- 
ramos anteriormente con respecto a la capacidad de recuperación de estos sujetos durante la tarea, que les llevó a establecer un control (mantenimiento) de su estado afectivo, sus recursos personales de carácter disposicional (optimismo, autoestima) pudieron intervenir favorablemente en la obtención de un rendimiento equiparable al del resto de los grupos.

En el caso de los pesimistas DB o interferidos hay que tener en cuenta que, a pesar de la ya referida inestabilidad afectiva intratarea, los niveles absolutos de ansiedad y afectividad eran adaptativos, no llegando a ser significativamente diferentes de los existentes en los pesimistas DA o congruentes. Esto supone que los pesimistas $\mathrm{DB}$, a pesar de haber sido interferidos, llegaron a la situación en unas condiciones adecuadas para afrontarla.

En otro lugar (Fernández, 1999; Fernández y Bermúdez, en prensa, $a, b)$ nos referimos a distintos factores que podrían dar cuenta de por qué en estudios pasados sobre el tema la interferencia ocasionada en la activación de estrategias en pesimistas defensivos ha provocado no sólo un deterioro afectivo, sino también en la ejecución, mientras que en nuestros estudios esto no ha ocurrido. Allí hacemos referencia a diferentes maneras de inducción de interferencia en ambos tipos de estudios podían estar en la base de ello, dando como resultado la instauración de un estado motivacional más favorable en nuestros sujetos que el existente en los pesimistas de aquellos estudios.

Finalmente, una explicación motivacional podría también utilizarse para intentar explicar la ausencia de niveles diferenciales de rendimiento entre los pesimistas DA asignados a una contingencia alta y a una baja. Independientemente de la cualidad del afrontamiento introducida por la creencia o no en la controlabilidad de un suceso ambiental (que lleva a diferentes estados afectivos), la alta motivación de logro (deseo de éxito) característica en estos sujetos (Fernández y Bermúdez, en prensa, $a, b$; Norem y Cantor, 1986a,b) ha podido compensar la limitación afectiva existente en los asignados a una contingencia baja, evitando así un deterioro en el rendimiento.

En futuros estudios sobre el tema se debería indagar más directamente en torno a este posible papel compensador de la motivación, procediendo a un análisis de la influencia de distintos niveles de implicación en la situación (motivación) y de su controlabilidad sobre la cualidad del afrontamiento desde el punto de vista afectivo y del nivel de rendimiento obtenido.

\section{REFERENCIAS BIBLIOGRÁFICAS}

Bruner, J.S., Goodnow, J.J., y Austin, G.A. (1956). A study of thinking. New York: Wiley.

Chapman, M., y Skinner, E.A. (1985). Action in development/ Development in action. En M. Frese y Sabini (Eds.), Goal directed behavior: The concept of action in psychology (pp.199-213). Hillsdale, NJ: Lawrence Erlbaum.

Conway, V.J., y Terry, D.J. (1992). Appraised controllability as a moderator of the effectiveness of different coping strategies: A test of the goodness of fit hypothesis. Australian Journal of Psychology, 44, 1-7.

Evans, G.W., Shapiro, D.H., y Lewis, M.A. (1993). Specifying dysfunctional mismatches between different control dimensions. British Journal of Psychology, 84, 255-273.

Fernández, E. (1999). Variables personales y situacionales en la autorregulación en contextos de logro: el caso del optimismo, el pesimismo defensivo y la controlabilidad de la tarea. Tesis doctoral no publicada. UNED, Madrid.

Fernández, E., y Bermúdez, J. (1999). Estructura factorial del Optimismo y el Pesimis- 
mo. Boletín de Psicología, 63, 7-26.

Fernández, E., y Bermúdez, J. (en prensa, a). Pesimismo defensivo, optimismo y dificultad de la tarea: el papel de las expectativas. Revista de Psicología General y Aplicada.

Fernández, E., y Bermúdez, J. (en prensa, $b$ ). Control situacional y focalización en la tarea en pesimistas defensivos y optimistas. Estudios de Psicología.

Folkman, S., Lazarus, R.S., Dunkel-Schetter, C., De Longis, A., y Gruen, R. (1986). Dynamics of a stressful encounter: Cognitive appraisal, coping, and encounter outcomes. Journal of Personality and Social Psychology, 50, 992-1003.

Forsythe, C.J., y Compas, B.E. (1987). Interaction of cognitive appraisals of stressful events and coping: Testing the goodness of fit hypothesis. Cognitive Therapy and Research, 11, 473-485.

Juilland, A., y Chang-Rodríguez, E. (1964). Frequency dictionary of Spanish words. London: Mouton.

Litt, M.D. (1988). Cognitive mediators of stressful experience: Self-efficacy and perceived control. Cognitive Therapy and Research, 12, 241-260.

Norem, J.K. (1989). Cognitive strategies as personality: Effectiveness, specificity, flexibility and change. En D.M. Buss y N. Cantor (Eds.), Personality Psychology: recent trends and emerging directions (pp. 45-60). New York: Springer-Verlag.

Norem, J.K., e Illinworth, K.S.S. (1993). Strategy-dependent effects of reflecting on self and tasks: Some implications for optimism and defensive pessimism. Journal of Personality and Social Psychology, 65, 822-835.

Norem, J.K., y Cantor, N. (1986a). Anticipatory and post-hoc cushioning strategies: Optimism and defensive pessimism in "risky" situations. Cognitive Therapy and Research, 10, 347-362.

Norem, J.K., y Cantor, N. (1986b). Defensive pessimism: Harnessing anxiety as motivation. Journal of Personality and Social Psychology, 51, 1208-1217.
Norem, J.K., y Cantor, N. (1990). Cognitive strategies, coping, and perceptions of competence. En R.J. Sternberg y J. Kolligian (Eds.), Competence considered (pp. 190204). New Haven, CT: Yale University Press.

Scheier, M.F., Carver, C.S. y Bridges, M.W. (1994).Distinguishing optimism from neuroticism (and trait anxiety, self-mastery, and self-esteem): A reevaluation of the Life Orientation Test. Journal of Personality and Social Psychology, 67, 1063-1078.

Scheier, M.F., y Carver, C.S. (1985). Optimism, coping and health: Assessment and implications of generalized outcome expectancies. Health Psychology, 4, 219247.

Showers, C., y Cantor, N. (1985). Social cognition: A look at motivated strategies. Annual Review of Psychology, 36, 275-305.

Spencer, S.M., y Norem, J.K. (1996). Reflection and distraction: Defensive pessimism, strategic optimism, and performance. Personality and Social Psychology Bulletin, 22, 354-365.

Spielberger, C.D., Gorsuch, R.L., y Lushene, R.E. (1970). State-Trait Anxiety Inventory (STAI). Palo Alto CA: Consulting Psychologists Press. (Versión española: Cuestionario de Ansiedad Estado-Rasgo, TEA Ediciones, Madrid, 1986).

Thoits, P.A. (1983). Dimensions of life events that influence psychological stress: An evaluation and synthesis of the literature. En H.B. Kaplan (Ed.), Psychosocial stress: Trends in theory and research (pp. 33-103). New York: Academic Press.

Watson, D., Clark, L.A., y Tellegen, A. (1988). Development and validation of brief measures of positive and negative affect: The PANAS scales. Journal of Personality and Social Psychology, 54, 1063-1070.

Weisz, J.R. (1983). Can I control it? The pursuit of veridical answers across the life span. En P.B. Baltes y O.G. Brim, Jr. (Eds.), Life-span development and behavior (pp. 233-300). New York: Academic Press. 


\section{APÉNDICE}

Elementos de la tarea de anagramas, y sus soluciones, clasificados por frecuencia de uso

\begin{tabular}{|c|c|c|c|c|c|}
\hline \multicolumn{2}{|c|}{ Baja frecuencia } & \multicolumn{2}{|c|}{ Media frecuencia } & \multicolumn{2}{|c|}{ Alta frecuencia } \\
\hline $\begin{array}{l}\text { ODMGA } \\
\text { TLAENET } \\
\text { UNFBEE } \\
\text { DEINTIOC } \\
\text { ENSDOTI } \\
\text { RPGEON } \\
\text { LPEIUGE } \\
\text { BTAIAR }\end{array}$ & $\begin{array}{l}\text { (DOGMA) } \\
\text { (LATENTE) } \\
\text { (FUNEBRE) } \\
\text { (IDENTICO) } \\
\text { (SENTIDO) } \\
\text { (PREGON) } \\
\text { (PLIEGUE) } \\
\text { (ABATIR) }\end{array}$ & $\begin{array}{l}\text { BIRLALR } \\
\text { ACGRAR } \\
\text { PAETTIO } \\
\text { RAORAJR } \\
\text { CAEIRTO } \\
\text { IALMGOR } \\
\text { CSALICO } \\
\text { NFIRIG } \\
\text { LADUIR } \\
\text { IDNETE }\end{array}$ & $\begin{array}{l}\text { (BRILLAR) } \\
\text { (CARGAR) } \\
\text { (APETITO) } \\
\text { (ARROJAR) } \\
\text { (ACIERTO) } \\
\text { (MILAGRO) } \\
\text { (CLASICO) } \\
\text { (FINGIR) } \\
\text { (ALUDIR) } \\
\text { (DIENTE) }\end{array}$ & $\begin{array}{l}\text { E DEBR } \\
\text { IT MEPO }\end{array}$ & $\begin{array}{l}\text { (DEBER) } \\
\text { (TIEMPO) }\end{array}$ \\
\hline
\end{tabular}

